\title{
GaAsP nanowires containing intentional and self-forming quantum dots
}

Fonseka, H. Aruni, Velichko, Anton, Zhang, Yunyan, Davis, George, Gott, James, et al.

H. Aruni Fonseka, Anton V. Velichko, Yunyan Zhang, George D. Davis, James A. Gott, Tillmann Godde, Ana M. Sanchez, Richard Beanland, Huiyun Liu, David J. Mowbray, "GaAsP nanowires containing intentional and self-forming quantum dots," Proc. SPIE 11291, Quantum Dots, Nanostructures, and Quantum Materials: Growth, Characterization, and Modeling XVII, 112910J (2 March 2020); doi: 10.1117/12.2543747

SPIE. Event: SPIE OPTO, 2020, San Francisco, California, United States 


\title{
GaAsP Nanowires Containing Intentional and Self-Forming Quantum Dots
}

\author{
H. Aruni Fonseka ${ }^{\mathrm{a}}$, Anton V. Velichko ${ }^{\mathrm{b}}$, Yunyan Zhang ${ }^{\mathrm{c}}$, George D. Davis ${ }^{\mathrm{b}}$, James A. Gott ${ }^{\mathrm{a}}$, \\ Tillmann Godde ${ }^{\mathrm{b}}$, Ana M. Sanchez $z^{\mathrm{a}}$, Richard Beanland ${ }^{\mathrm{a}}$, Huiyun Liu ${ }^{\mathrm{c}}$ and David J. Mowbray ${ }^{\mathrm{b}}$ \\ ${ }^{a}$ Department of Physics, University of Warwick, Coventry CV4 7AL, United Kingdom. \\ ${ }^{b}$ Department of Physics and Astronomy, University of Sheffield, Sheffield S3 7RH, United \\ Kingdom. \\ ${ }^{c}$ Department of Electronic and Electrical Engineering, University College London, London WC1E \\ 7JE, United Kingdom.
}

\begin{abstract}
GaAsP nanowires (NWs) containing a range of different heterostructures are shown to be a highly promising system for the fabrication of efficient and novel ultra-small light emitters. NWs containing GaAs radial quantum wells (QWs) have emission with high thermal stability, due to both large electron and hole confinement potentials. A structure containing three QWs exhibits very low threshold lasing at low temperatures. Within the GaAsP central region of the same NW, the formation of quantum wires (QWRs) on three of the six vertices is observed, these QWRs are aligned parallel to the NW axis. The presence of twins causes a $180^{\circ}$ rotation of the crystal about the growth axis, breaking the QWRs into short sections which may act as quantum dots (QDs). Optical studies of the NWs support the formation of optically active QWRs and QDs. In a second type of NW, during growth of the GaAsP NW core the introduction of a short GaAs section forms a QD. The inclusion of up to 50 QDs with high structural and optical quality is shown to be possible; indicating the potential for the fabrication of QD lasers. A structure with only one QD exhibits a single sharp emission line and behavior consistent with single exciton recombination. The addition of passivation layers, grown as a shell on the NW core, is shown to be essential in obtaining good optical properties. Our studies hence demonstrate that GaAsP-GaAs NWs containing heterostructures have significant potential for a range of novel light emitting applications.
\end{abstract}

Keywords: Nanowires, GaAsP, lasers, quantum wells, quantum dots, quantum wires, III-V

\section{INTRODUCTION}

Semiconductor nanowires (NWs) provide a very different architecture in comparison to more common thin film growth ${ }^{1,2,3}$. Advantages include a much larger surface area, reduced material consumption, the intrinsic formation of an optical cavity, placement at predefined positions, and the ability to incorporate high levels of strain without the formation of dislocations. The latter allows increased choice of material combinations and growth on a wider range of substrates, for example III-V NWs on $\mathrm{Si}^{4}{ }^{4,5}$. Whilst initial work concentrated on the growth and study of pure NWs, more recently there has been significant interest in the incorporation of heterostructures for increased functionality ${ }^{7}$. For example, radial quantum wells $(\mathrm{QWs})^{8}$ or axial quantum dots (QDs) ${ }^{9}$ have been added to NWs and employed as both ultra-small lasers ${ }^{4}$ and non-classical light emitters ${ }^{10,11}$. In addition, observation of unintentional heterostructures, including both QDs ${ }^{12,13,14,15,16}$ and quantum wires (QWRs) ${ }^{15}$ forming on the vertices of NWs, has been reported.

There are a number of requirements for optimized performance when designing NW heterostructures for light emission applications. These include large electron and hole confinement energies, the absence of a central core having a lower band gap than the emitting region, which otherwise acts as both an absorbing region and carrier reservoir, low defect densities and the ability to add effective surface passivation. In this paper, we show that the GaAsP-GaAs system is a very promising candidate for the growth of high structural and optical quality NWs containing heterostructures for application as photon emitters. Multiple radial GaAs QWs can be incorporated within GaAsP NWs and demonstrate lasing at very low pump powers. QWRs and QDs form spontaneously during the growth of the central region of GaAsP NWs. These QDs appear to be unique to the current system and arise from the formation of QWRs on only three of the six vertices and the influence of crystal twins. Finally, intentional GaAs QDs can be added to the core of the GaAsP NWs by switching the sources during growth. Surface passivation layers, added as shells to the core, are found to be necessary to achieve high optical quality. 


\section{EXPERIMENTAL RESULTS}

\subsection{Growth and Experimental Details}

GaAsP NWs were grown without the use of a foreign metal catalyst, instead using native Ga droplets. All samples were grown by solid-source MBE on p-type $\mathrm{Si}(111)$ substrates ${ }^{17}$. The basic radial QW structure, containing a single radial well, was based on a $\mathrm{GaAs}_{0.62} \mathrm{P}_{0.38}$ core, grown for $1.5 \mathrm{hr}$ at a temperature of $\sim 640^{\circ} \mathrm{C}$. Following the core growth, the Ga droplet was consumed and growth changed from axial to radial. Two radial GaAs $0.54 \mathrm{P}_{0.46}$ shells were grown separated by a $\sim 10$ $\mathrm{nm}$ GaAs QW. No surface passivation was added to this sample. To achieve lasing additional gain material is required, and for this purpose NWs containing three QWs were grown. Here the core consists of $\mathrm{GaAs}_{0.38} \mathrm{P}_{0.62}$ and the active region consists of three GaAs QWs, separated by $\mathrm{GaAs}_{0.53} \mathrm{P}_{0.47}$ barriers. To passivate the surface additional $\mathrm{Al}_{0.5} \mathrm{Ga}_{0.5} \mathrm{As}_{0.53} \mathrm{P}_{0.47}$ and $\mathrm{GaAs}_{0.53} \mathrm{P}_{0.47}$ shells were added. The diameters of the single and three QW containing NWs were 130 and $400 \mathrm{~nm}$, respectively, and typical NW lengths were 5-6 and 9-10 $\mu \mathrm{m}$, respectively ${ }^{18},{ }^{19}$. Structures with intentional axial QDs were fabricated by switching the P source off to form GaAs for a short section during the GaAsP core growth. Here the height of the QD is determined by the GaAs growth time and the lateral dimensions by the diameter of the NW core. Samples with either one or fifty QDs were grown. Samples for structural studies consisted of just the core but for optical studies it was found necessary to add similar surface passivation layers as those used for the radial QW containing structures.

Transmission electron microscopy (TEM) structural measurements were performed on NWs mechanically transferred onto a holey carbon support. Samples for cross-sectional TEM were obtained by microtome slicing of NWs embedded in low viscosity (LV) resin. TEM measurements were obtained using JEOL 2100 and doubly corrected ARM200F microscopes, both operating at $200 \mathrm{kV}$.

Micro-photoluminescence ( $\mu$-PL) measurements of single NWs were obtained following mechanical transfer and spatial dispersal on a new Si-wafer with the NW axis parallel to the wafer surface. PL was excited with a $515 \mathrm{~nm}$ continuous wave (cw) diode laser, focused to a spot size of $\sim 1 \mu \mathrm{m}$ diameter with a 20x long working distance microscope objective. Measurements were performed with samples in a He cryostat with a base temperature of $6 \mathrm{~K}$. The emitted PL was dispersed by a $0.75 \mathrm{~m}$ spectrometer and detected with a liquid-nitrogen cooled CCD. The high intensity pumping required to achieve lasing action was provided by a pulsed $640 \mathrm{~nm}$ diode laser with a pulse length of $80 \mathrm{ps}$ and repetition frequency of 80 MHz. The incident laser light was focused to a line $\left(30 \times 1.4 \mu \mathrm{m}^{2}\right)$ using a cylindrical lens to ensure photo-pumping of the whole NW.

\subsection{GaAsP NWs containing GaAs QWs}

Figure 1 shows a typical $\mu$-PL spectra at $6 \mathrm{~K}$ for the NW containing a single GaAs radial QW. At long wavelengths $(\sim 740$ $\mathrm{nm}$ ) the intense emission is due to the QW. The weaker emission in the range $\sim 610-670 \mathrm{~nm}$ arises from the GaAsP barrier and is discussed in detail in the following section. With increasing temperature, the intensity of the QW emission decreases as carriers are thermally excited out of the QW. From macro-PL measurements of a large number of NWs on the original substrate, a large activation energy of $125 \pm 12 \mathrm{meV}$ for the quenching of the PL is determined. Simulations of the electronic structure using nextnano software ${ }^{20}$ give separations between the lowest energy QW electron and hole states and relevant barrier band edges of $\sim 115$ and $300 \mathrm{meV}$, respectively. Hence, the measured activation energy is close to the smaller of these two separations, which is that of the electrons. In the current structures both energies determining electron and hole loss from the QW are relatively large $(\sim 115$ and $300 \mathrm{meV})$. This is a significant advantage for devices required to operate at elevated temperatures and is a result of the mixed group- $\mathrm{V}$ nature of the GaAsP-GaAs system and the large compressive strain applied to the GaAs QW which further increases confinement. 


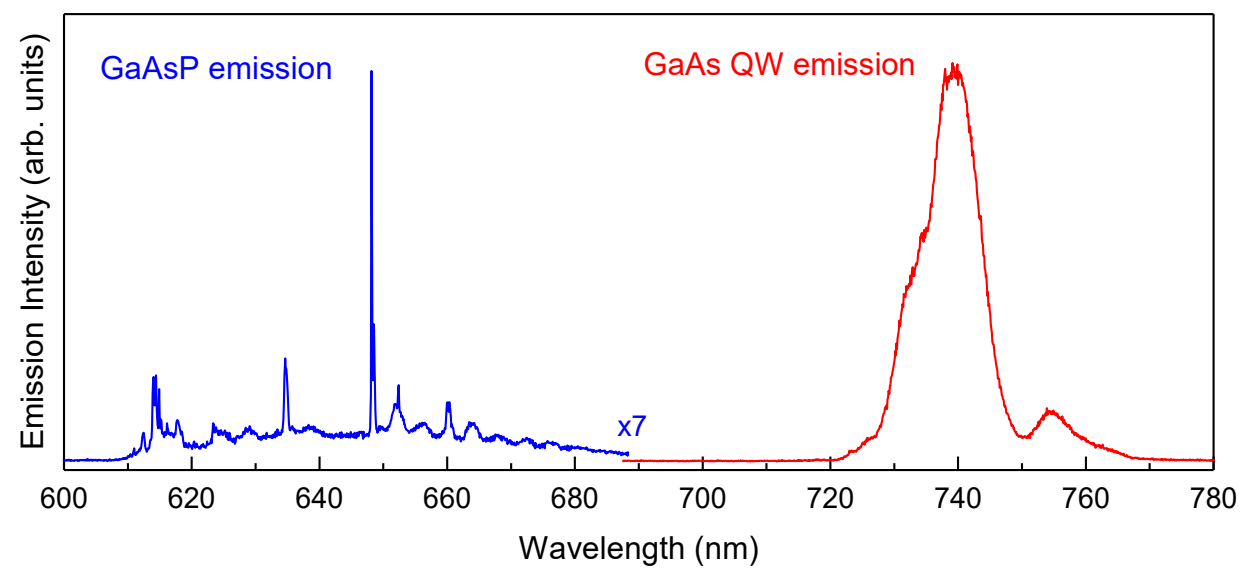

Figure 1. 6K $\mu$-PL spectra of a single GaAsP NW containing a single GaAs QW.

To obtain lasing from a NW with a QW active region, more than one QW is required to achieve sufficient gain. Thus, a sample was grown with three nominally identical QWs and with additional surface passivation layers. Figure 2 (a) shows a cross section annular dark field (ADF) scanning transmission electron microscopy (STEM) image with an overlaid energy dispersive x-ray (EDX) map of P from a NW with this structure. The hexagonal NW cross section and the three GaAs QWs are clearly visible. Figure 2 (b) shows the composition profile for As, P and Ga elements along the arrow indicated in Figure 2 (a). The QWs exhibit a width inhomogeneity, which is caused in part by shadowing effects from randomly positioned surrounding NWs. Improved uniformity should be achievable by growing on pre-patterned substrates to ensure an increased and uniform spacing between NWs. Even though the strain of the QW is relatively high $(\sim 1.7 \%)$, there is no evidence of strain-induced line or loop-type dislocations.
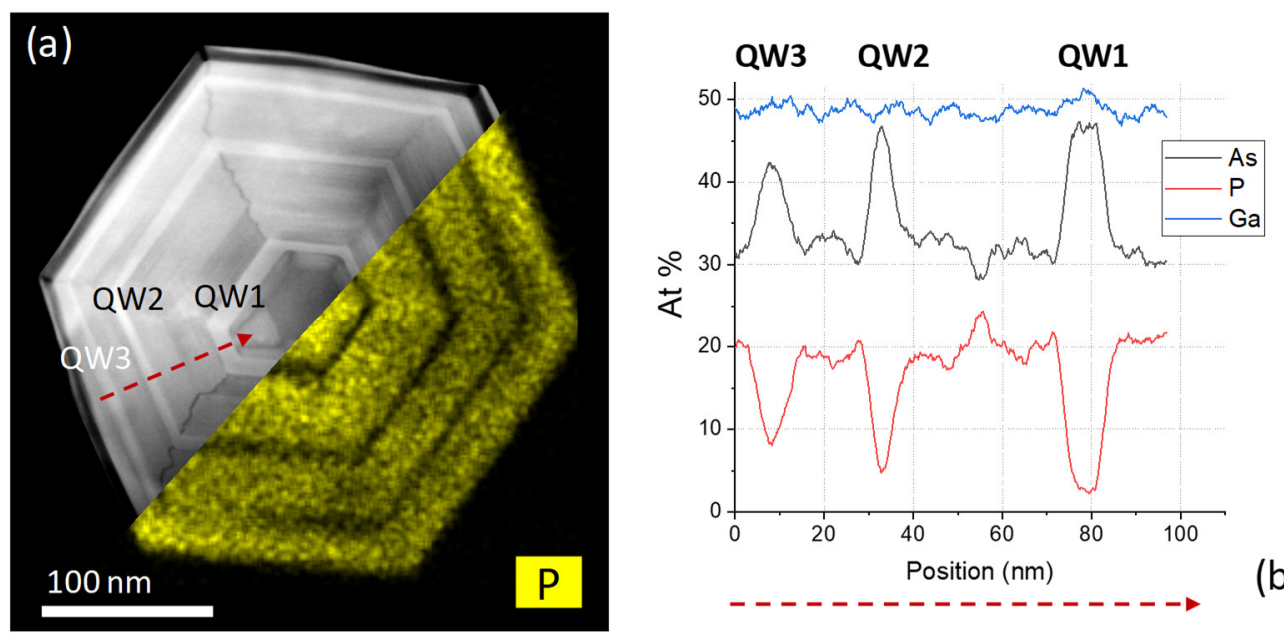

Figure 2. (a) Cross sectional ADF STEM image of a GaAsP NW containing three radial GaAs QWs. The lower right hand side shows the $\mathrm{P}$ elemental map. The QWs are visible as the pale regions (darker regions in the elemental map) and the AlGaAsP appears as the outer darker band. (b) Compositional profiles for $\mathrm{Ga}$, As and $\mathrm{P}$ recorded along the arrow indicated in (a).

Figure 3 (a) shows QW emission spectra recorded at different positions along the length of a NW containing three QWs. The emission is relatively uniform, although there is some sub-structure which may indicate the presence of different well widths, as in Figure 2. No emission is observed for excitation at one end of the NW, this is attributed to the presence of defects in the NW tip ${ }^{21}$. Figure 3 (b) shows power dependent $\mu$-PL spectra for excitation in the middle region of a NW. The QW emission exhibits a broadening towards higher energy with increasing laser power, consistent with carrier state filling of the QW. 

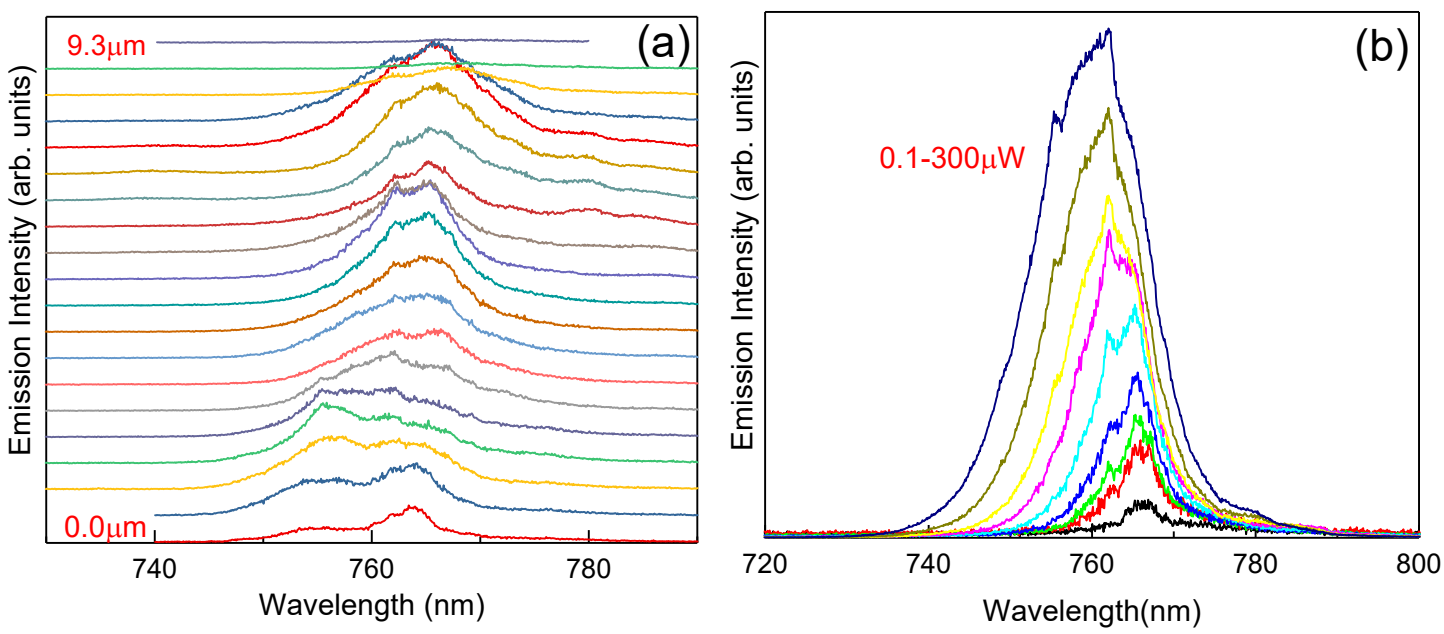

Figure 3. (a) $6 \mathrm{~K} \mu$-PL spectra recorded at different positions along the axis of a GaAsP NW containing three GaAs QWs. The spectral window corresponds to the emission from the QW. (b) Power dependent $\mu$-PL spectra of the QW emission.

To achieve lasing, higher carrier densities are required than that can be provided by a cw laser. Therefore, a pulsed laser was used which is able to provide significantly higher peak powers and hence larger excited carrier densities. Figure 4 (a) shows spectra recorded for increasing pump power and for a sample temperature of $6 \mathrm{~K}$. Initially there is a further statefilling shift to higher energy followed by the emergence of Fabry-Pérot cavity modes. At the highest pump powers, the intensities of a small number of cavity modes increases super-linearly, indicating the onset of lasing. Figure 4 (b) plots the intensity of the strongest mode as a function of pump pulse energy density, the pump fluence. At $6 \mathrm{~K}$ the data indicates a lasing onset of $\sim 20 \mu \mathrm{J} / \mathrm{cm}^{2} /$ pulse. With increasing temperature the threshold increases, reaching a value of $\sim 50 \mu \mathrm{J} /$ $\mathrm{cm}^{2} /$ pulse at $100 \mathrm{~K}$. No lasing was observed at higher temperatures as a result of the threshold exceeding the maximum pump power of the current laser.
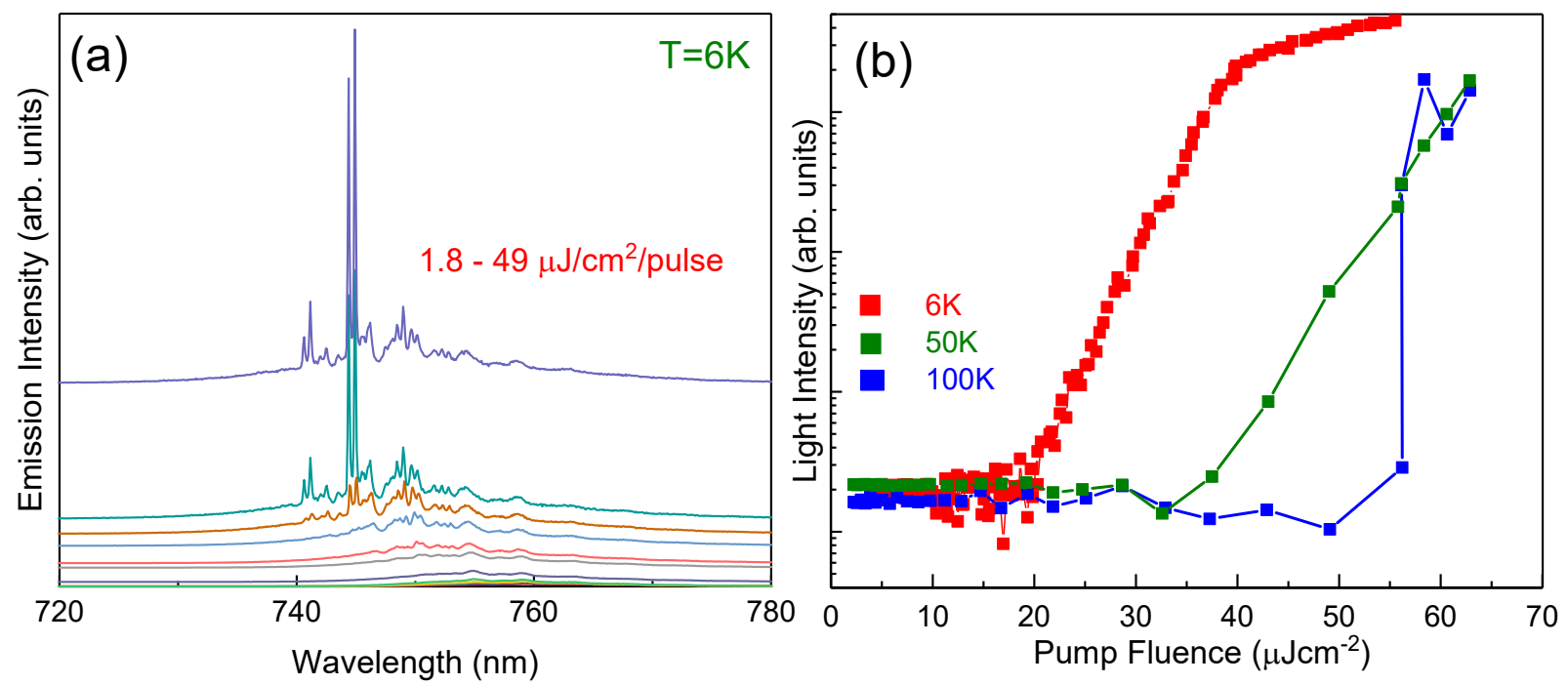

Figure 4. (a) $\mu$-PL power dependent spectra for high carrier densities at $6 \mathrm{~K}$. (b) Pump fluence dependence of the intensity of the strongest cavity mode at three temperatures: 6,50 and $100 \mathrm{~K}$.

Comparison with previous reports of lasing from NWs, containing either QWs or QDs, is complicated by the different experimental conditions used, particularly the excitation wavelength and the extent to which this is absorbed by the sample. In the current experiments the red pump laser has a photon energy below the GaAsP barrier bandgap and so only weak, direct absorption by the three GaAs QWs occurs (estimated at $\sim 5 \%$ ). Available previously reported values include: 19 
$\mu \mathrm{J} / \mathrm{cm}^{2} /$ pulse at $80 \mathrm{~K}$ for a AlGaAs $/$ GaAs single QW $22,400 \mu \mathrm{J} / \mathrm{cm}^{2} /$ pulse at $10 \mathrm{~K}$ for a GaAs-InGaAs multiple QW ${ }^{23}, 43$ $\mu \mathrm{J} / \mathrm{cm}^{2} /$ pulse at $300 \mathrm{~K}$ for a GaAs-AlGaAs multiple $\mathrm{QW}^{24}$ and $25 \mu \mathrm{J} / \mathrm{cm}^{2} /$ pulse at $7 \mathrm{~K}$ for a GaInAs-GaAs QD structure ${ }^{25}$. Hence, the current results are competitive with previous reports, with the exception of the highly optimized lasers of Ref 24. Further significant improvement of the nanowires is expected via additional growth optimization. This could include the removal of defects from the tip of the NW, which may act as non-radiative recombination centers, improving the QW homogeneity by growing the NWs on pre-patterned substrates, improving the Ga droplet removal, which is believed to result in a poor quality upper facet, and optimization of the number and placement of the QWs. In addition to the strong electron and hole confinement, which should aid high temperature operation, the current system has the additional advantage of high compressively strained (estimated to be $\sim 1.7 \%$ ) QWs. For planar lasers compressive strain has been shown to be advantageous in increasing the heavy-light hole separation and modifying the in-plane hole mass, allowing a given gain to be achieved at a lower carrier density ${ }^{26}$. Furthermore, the GaAs QWs, which act as the gain material, represents the lowest band gap region in this system. This is advantageous compared to material systems such as AlGaAsGaAs, which has a core with a lower bandgap than that of the gain region. In this case the core acts as both a carrier sink and photon absorbing region, both these loss mechanisms will degrade device performance.

\subsection{Self-Forming Quantum Wires and Quantum Dots in GaAsP Nanowires}

Figure 5 (a) shows an ADF STEM cross sectional image of a GaAsP NW containing a single GaAs QW. Similar to Figure 2(a), the regions with a higher P composition appear darker. Clearly visible is the single radial GaAs QW which appears as a paler band.
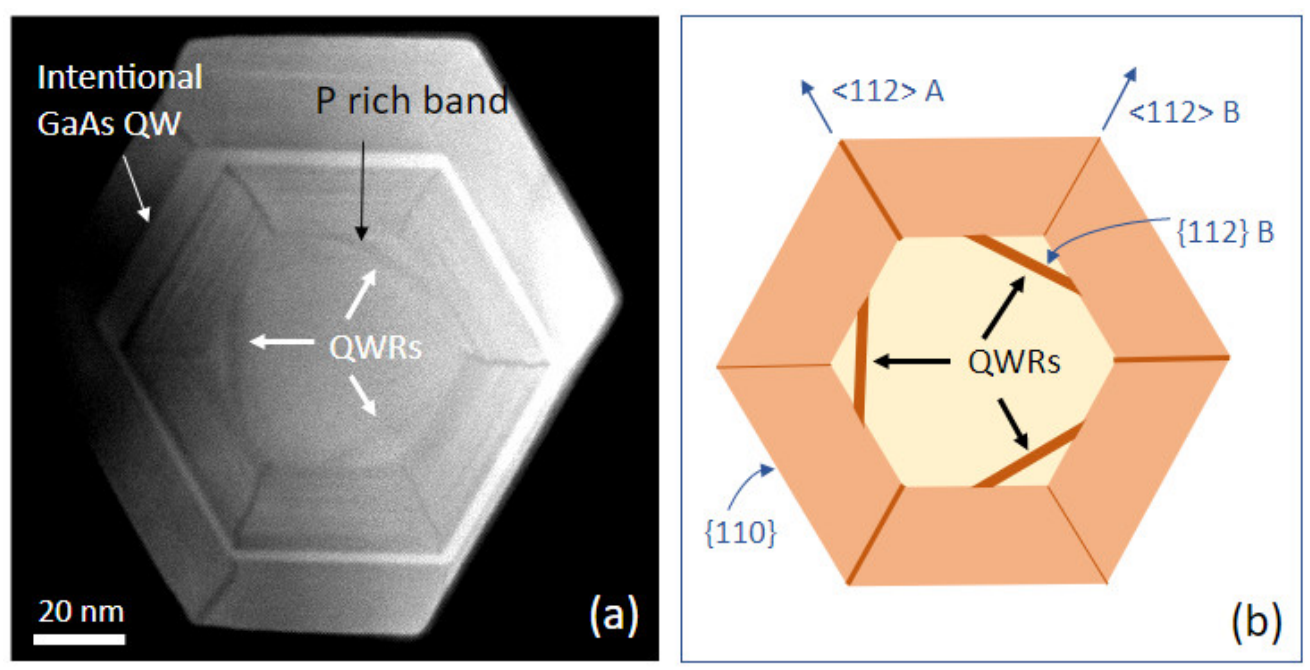

Figure 5. (a) ADF-STEM cross sectional image of a GaAsP NW containing a single radial GaAs QW. Self-formed structures within the nominally uniform GaAsP core and shell results in the formation of three QWRs running parallel to the axis of the NW. (b) Schematic diagram showing the main structure of the QWRs and relevant crystallographic directions.

Within the central region of the NW are triangular regions, formed at three of the six vertices of the NW. These have a lower $\mathrm{P}$ content than the surrounding, darker GaAsP regions which are formed by shell growth on two sides and a $\mathrm{P}$ rich band on the inner side. Detailed analysis reveals that these three regions are formed along $<112>\mathrm{B}$ directions, as indicated in the schematic of Figure 5 (b). Similar triangular shaped regions are present in images recorded from closely spaced points along the length of the same NW, confirming that these features run along the length of the NW. Compositions extracted from EDX measurements confirm that the triangular regions have a lower P composition than the surrounding material. As the band gap of GaAsP increases with $\mathrm{P}$ content, the triangular regions form a lower bandgap structure embedded in a higher bandgap matrix, and hence may act as QWRs running parallel to the axis of the NW. The compositions of the different regions exhibit significant inter- and intra-NW variations. Whilst the $\mathrm{P}$ composition of the shell is fairly constant at $\sim 47 \%$, the P composition of the core varies between $\sim 35$ and $43 \%$ and that of the triangular region (henceforth referred to as a QWR) varies between $\sim 30$ and $45 \%$. Calculations were performed using nextnano ${ }^{20}$ 
software for a range of parameters for the core, QWR and P rich-band compositions and QWR height. These calculations demonstrate that for most combinations, the QWR is able to localise both electrons and holes, and hence potentially behave as an optically active QWR.
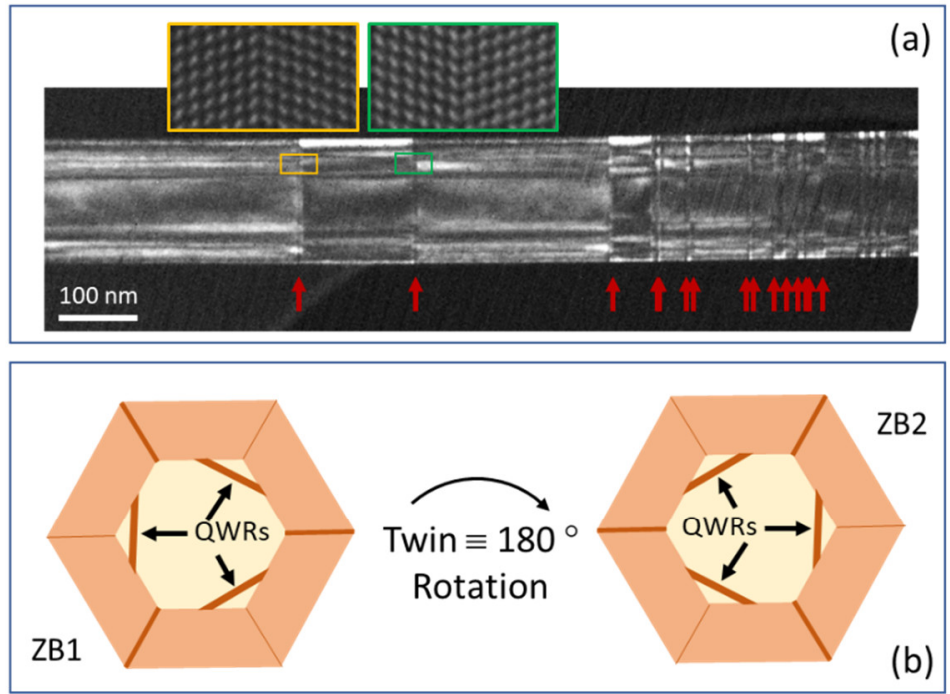

(c)

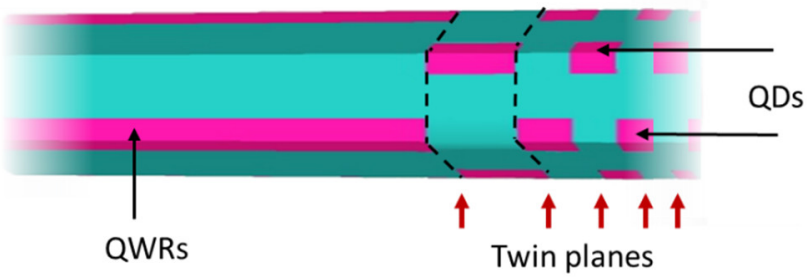

Figure 6. (a) TEM image showing the presence of twin boundaries which start to appear in the middle third of the NW and increase in frequency towards the tip. (b) Schematic diagram showing the effect of a twin on the structure of a [111] NW. The twin results in a $180^{\circ}$ rotation of the crystal around the [111] axis. (c) Schematic showing the formation of QDs via twinning of the QWR.

Structural studies (Figure 6 (a)) reveal twin boundaries which start in the middle third of the NW and increase in frequency towards the NW tip. A twin which is normal to the [1 111$] \mathrm{NW}$ growth direction produces a $180^{\circ}$ rotation of the crystal, as shown in Figure 6 (b). As QWRs only occur on three of the six NW vertices, this has the effect of breaking up the QWRs into short sections, as shown in Figure 6 (c). These short QWR segments can act as optically active QDs if two conditions are satisfied. First, the triangular regions must be sufficiently small that when rotated there is no spatial overlap between segments from the adjoining twin, and second, the core P content must be higher than that of the QWRs in order to provide three-dimensional carrier confinement. If these conditions are satisfied then the short QWR segments may act as QDs, with carrier confinement in all three spatial directions. nextnano simulations indicate that for an $8 \mathrm{~nm}$ high QD the additional confinement compared to that of the QWR is of order $90 \mathrm{meV}$.

Figure 7 shows $6 \mathrm{~K} \mu$-PL spectra recorded at regular intervals along the axis of a NW which showed emission when excited around $1-2 \mu \mathrm{m}$ from the base and for the spectral region corresponding to the GaAsP emission. The emission consists of a relatively small number of sharp lines (linewidths as low as $\sim 80 \mu \mathrm{eV}$ are observed), whose relative intensities vary with the position of the exciting laser. The most consistent and strongest emission occurs for the line at $648 \mathrm{~nm}$, this is observed along the whole length of the NW. The intensities of lines to longer wavelengths show a similar positional dependence, indicating a common origin. Lines to shorter wavelengths are typically only observed when exciting close to the central region of the NW and exhibit a stronger variation in intensity with respect to excitation position. There is also a weak, but broad, background between $\sim 630-700 \mathrm{~nm}$ which may arise from the GaAsP NW core. Emission is weaker towards both the base and the tip of the NW, probably a result of non-radiative recombination at the broken and defective ends of the NW. 


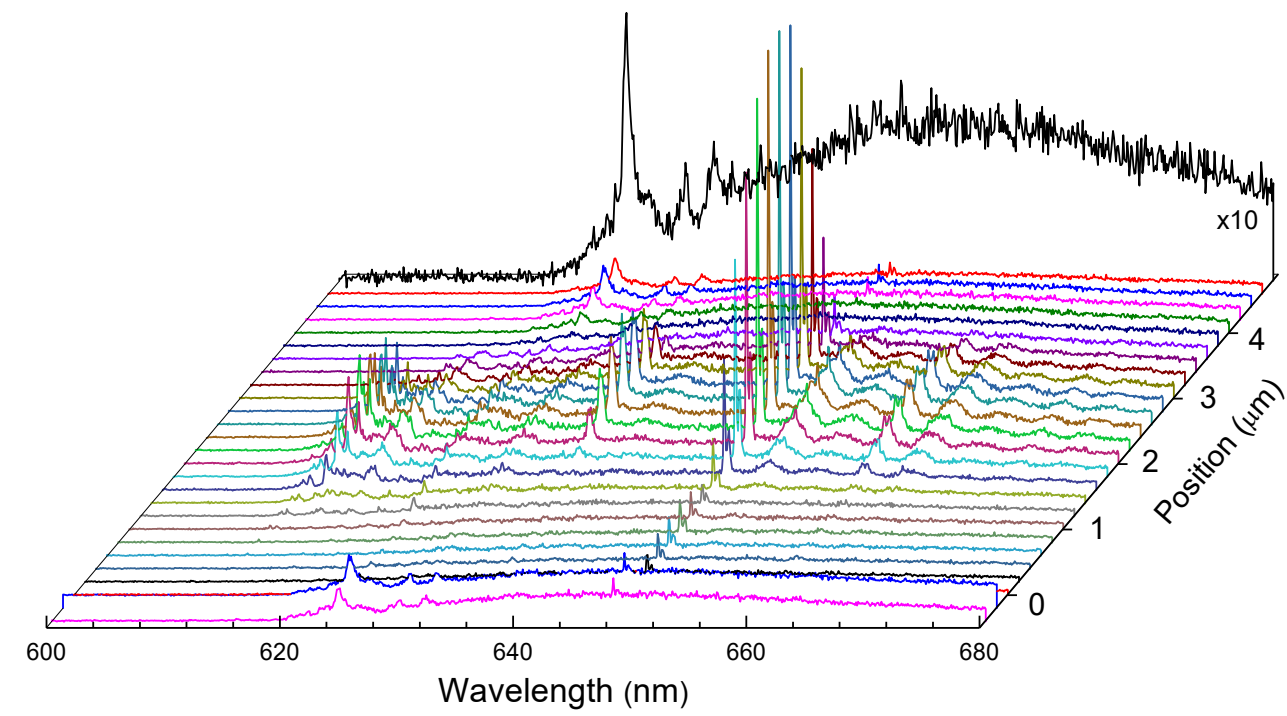

Figure 7. $6 \mathrm{~K} \mu$-PL spectra recorded for different positions along the NW axis for the spectral region corresponding to the GaAsP emission. The final spectrum has been magnified to show more clearly the broad emission between $\sim 630-700 \mathrm{~nm}$.
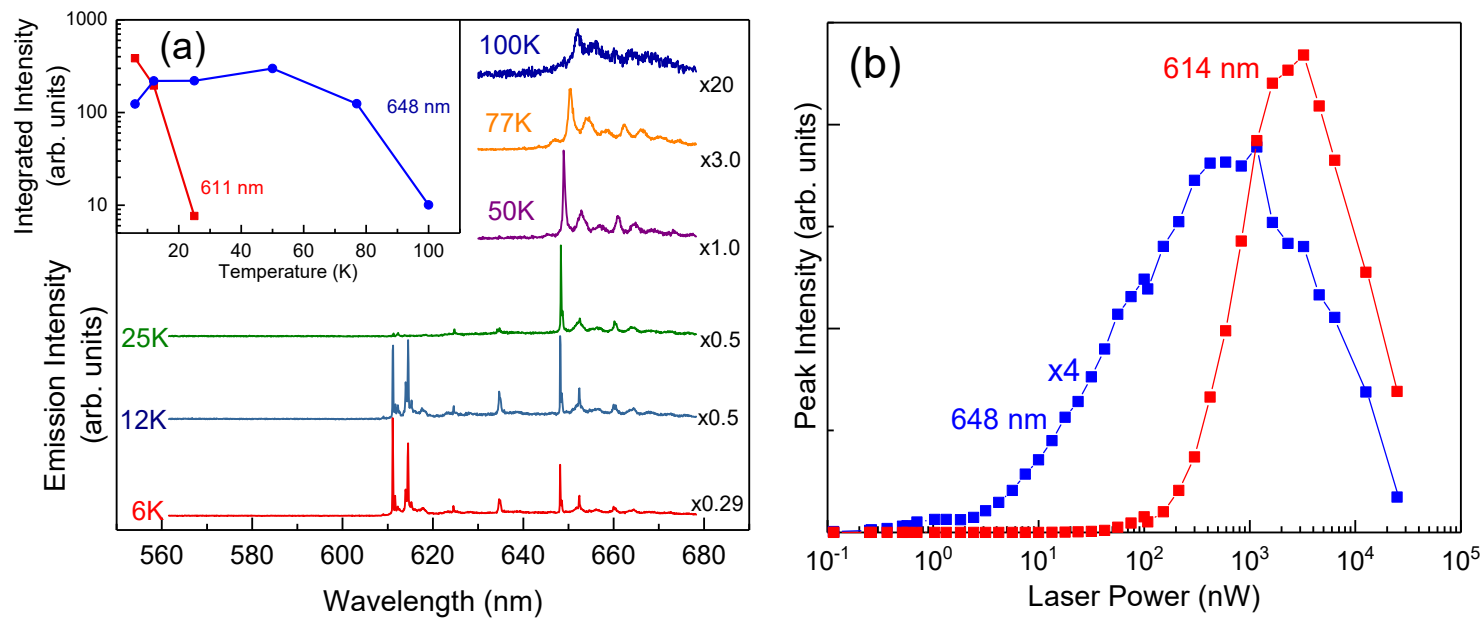

Figure 8. (a) Temperature dependent $\mu$-PL spectra. The inset plots the integrated intensity of two representative lines from the spectral regions $\sim 650 \mathrm{~nm}$ and $\sim 615 \mathrm{~nm}$. (b) Power dependence of the emission intensities of two of the sharp lines observed in the spectra of Figure 7.

Figure 8 (a) show $\mu$-PL spectra recorded as a function of temperature over the range 6 to $100 \mathrm{~K}$. In the inset the intensities of two representative lines, one at short the other at longer wavelength are plotted against temperature. The intensity of lines at $640 \mathrm{~nm}$ and above are relatively insensitive to temperature, still being observed at $100 \mathrm{~K}$. In contrast, lines below $640 \mathrm{~nm}$ quench fully by $25 \mathrm{~K}$. In addition, the emission of the longer wavelength lines is found to be polarized along the $\mathrm{NW}$ axis, whereas the shorter wavelength lines can show a linear polarization axis inclined by as much as $30^{\circ}$ to the NW axis.

Figure 8 (b) plots the intensities of the strongest emission line at $648 \mathrm{~nm}$, and a shorter wavelength line at $614 \mathrm{~nm}$, as a function of incident laser power. In common with the majority of lines, the emission at $648 \mathrm{~nm}$ shows an initial ( $\lesssim 3 \mathrm{nW})$ linear increase with power, followed by a saturation and then a decrease at high powers. This behavior is consistent with single exciton emission from a QD-like state ${ }^{27}$. In contrast, some lines exhibit a quadratic or higher power variation (the $614 \mathrm{~nm}$ exhibits an initial power dependence with an exponent of 2.3), consistent with higher order excitonic processes ${ }^{27}$. 
The above optical data suggests the presence of two distinct types of emitting center arising from the GaAsP. Emission at $640 \mathrm{~nm}$ and above occurs for excitation along the majority of the NW, is relatively insensitive to temperature and is polarized along the NW axis. In contrast, shorter wavelength emission is only observed for excitation in the middle region of the NW, is much more sensitive to temperature and has a more complex polarization behavior. The longer wavelength emission is hence attributed to the QWRs and the shorter wavelength emission to the QDs formed by the twinning induced truncation of the QWRs. The spectral separation between the two groups of emission lines is $\sim 30-50 \mathrm{~nm}(\equiv \sim 90-150 \mathrm{meV})$, consistent with the difference between the calculated ranges of QD-QWR emissions for structures with typical compositions and dimensions experimentally observed. The fact that the emission attributed to the QWRs demonstrates QD-like excitation power dependent behavior may be explained by the presence of compositional and/or size fluctuations along the QWR axis, that result in a series of elongated segments which exhibit quasi-QD properties.

Related to the current observation of triangular QWRs formed within GaAsP NWs is the observation of six QWRs formed in wurtzite GaN-AlN NWs ${ }^{28}$. In this system, QWRs form on all six identical vertices of the hexagonal NWs. In the current system QWRs form in only three of the six $<112>$ directions, corresponding to the subset of $<112>\mathrm{B}$ directions of the zinc-blende crystal structure. This indicates a variation in growth between the $<112>\mathrm{A}$ and $\mathrm{B}$ different polar surfaces, which may include differences in growth rate, decomposition rate and diffusion lengths. Because QWRs are only formed on three of the six directions, this allows twins to break the QWRs into short sections, giving rise to the formation of QDs. Self-assembling QDs have been observed in other NW systems ${ }^{12,13,14,15,16,29,30}$ although formed by very different mechanisms.

\subsection{Axial GaAs QDs in GaAsP NWs}

By modulating the epitaxial growth during the formation of the NW core it is possible to introduce short GaAs segments into the GaAsP NW. If both the diameter of the core and the height of the GaAs are sufficiently small, the GaAs regions will form QDs. Unlike self-assembled growth, this technique gives greater control over the shape and size of the QDs, and the possibility to stack large numbers of essentially identical dots, which is a requirement for laser applications. To investigate the feasibility of stacking a large number of QDs, a sample was grown consisting of 50 GaAs dots in a $\mathrm{GaAs}_{0.6} \mathrm{P}_{0.4} \mathrm{NW}$. Figure 9 shows a TEM image of a section of a NW from this sample. The NW has a diameter of $\sim 50 \mathrm{~nm}$ and the height of the QDs varies from 23 to $38 \mathrm{~nm}$ from the base to tip of the NW. The separation between QDs also increases from base to tip, with an average value $\sim 150 \mathrm{~nm}$. By modifying the growth conditions along the NW it will be possible to improve the uniformity of the QDs. The QDs are well defined and the interfaces between the GaAs and GaAsP are found to be quite sharp, with widths of $\sim 6-13$ monolayers.

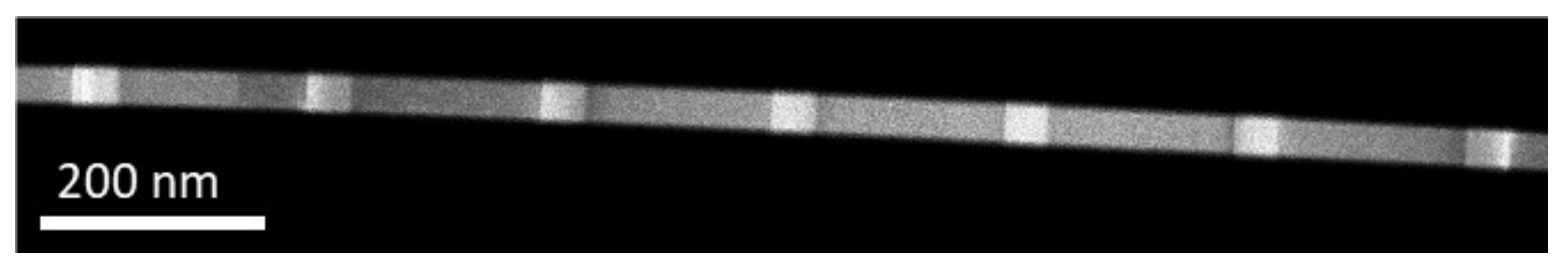

Figure 9. ADF STEM image of a section of a GaAsP NW containing 50 GaAs QDs.

Figure 10 (a) shows $\mu$-PL spectra for a structure similar to that of Figure 9, but with the addition of passivation layers grown around the NW core. The 50 QDs extend along a distance $\sim 9 \mu \mathrm{m}$ and the total NW length is $\sim 12-13 \mu \mathrm{m}$. The $1 \mu \mathrm{m}$ diameter laser spot excites a minimum of 7 QDs, although this number could be significantly larger as a result of carrier diffusion along the NW axis. Relatively intense emission is observed in the region $\sim 710-770 \mathrm{~nm}$ which is attributed to the QDs. Strong QD emission is observed as the laser spot is moved over a distance $\sim 9 \mu \mathrm{m}$, consistent with the known spatial distribution of the QDs. The observation of strong PL over the entire QD region indicates that the majority of dots are optically active and that there is no increase in defects as successive dots are grown. This is in agreement with detailed structural studies of the NWs without passivation, which show $66 \%$ of the dots to be defect free, with the remaining dots containing small numbers of twinning planes, which are not expected to impact their radiative efficiency. 

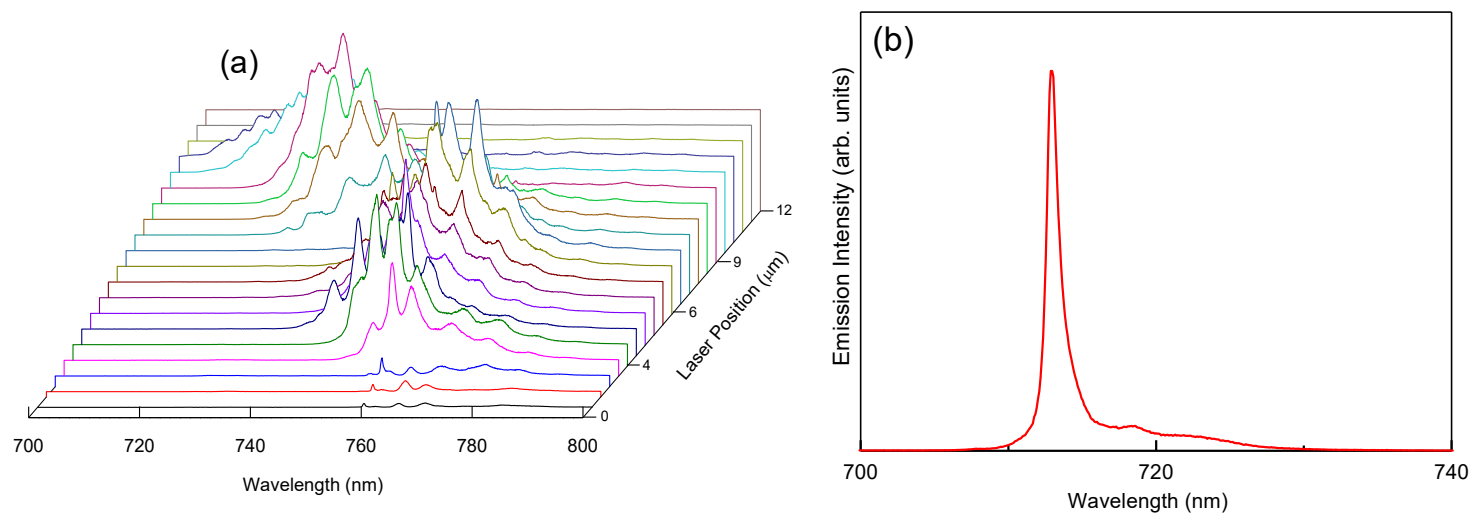

Figure 10. (a) $6 \mathrm{~K} \mu$-PL spectra recorded at different positions along the length of a GaAsP NW containing 50 GaAs QDs. (b) Spectrum of a similar structure containing a single QD.

The 50 QDs are not sufficiently separated to probe the properties of individual dots given that the minimum spatial resolution of the $\mu$-PL is $\sim 1 \mu \mathrm{m}$. To observe a clear QD signature it is necessary to study a single dot. Figure 10 (b) shows a $6 \mathrm{~K} \mu$-PL spectrum of a $\mathrm{GaAs}_{0.6} \mathrm{P}_{0.4} \mathrm{NW}$ containing only one GaAs QD. The NW diameter is $\sim 40 \mathrm{~nm}$ and the QD height is $\sim 25 \mathrm{~nm}$. The spectrum is recorded for low excitation power, such that the carrier occupancy of the dot is very low $(<<1)$. A relatively sharp, single emission line is observed, representing single excitonic recombination. To obtain this sharp emission, it is found necessary to add surface passivation layers to the NW core which minimize the influence of surface states on the QD. Linewidths as small as $500 \mu \mathrm{eV}$ are achievable at $6 \mathrm{~K}$. Power dependent $\mu$-PL measurements demonstrate the expected behavior for excitonic emission, showing an initial linear increase with increasing power followed by saturation and then reducing intensity at high powers ${ }^{27}$. With increasing laser power additional lines, which show a stronger power dependence (quadratic or higher), appear, consistent with multi-exciton processes ${ }^{27}$. There is hence clear evidence for the formation of an optically active QD. As was found for the GaAsP-GaAs QW structures, the PL intensity is relatively stable with increasing temperature, a result of the large electron and hole QD confinement potentials. This further demonstrates the suitability of the GaAsP-GaAs system as the basis for ultra-small and novel light emitters operating at elevated temperatures.

\section{CONCLUSIONS}

GaAsP NWs have been shown to form high quality optical systems, suitable for novel light emitters operating at elevated temperatures. Radial GaAs QWs can be added to the NWs to form the gain region of lasers. A structure with three QWs exhibits a very low lasing threshold at low temperatures ${ }^{18}$. Advantages of this system include deep electron and hole confinement potentials and a high compressive strain applied to the GaAs QWs. QWRs and QDs are shown to form spontaneously between the core and the initial shell of the GaAsP NWs ${ }^{19}$. In contrast to previous reports, the QWRs form on only three of the six vertices. This allows the truncation of the QWRs to form QDs via the axial twin boundaries which rotate the crystal through $180^{\circ}$. Intentional QDs can be added to the NW core by modulating the epitaxial growth. Unlike self-assembled QDs there is greater control of the dot size and shape, and stacking of large numbers of dots is possible. A structure with 50 optically active dots is demonstrated. Studies of a structure containing a single QD confirm the expected optical behavior. The GaAsP-GaAs NW system therefore has significant potential for novel ultra-small electro-optic devices operating well above liquid-nitrogen temperatures.

\section{ACKNOWLEDGEMENTS}

We acknowledge the support of the UK Engineering and Physical Sciences Research Council (EPSRC) via grants EP/P000916/1, EP/P000886/1, EP/006973/1 and EP/N509796/1. The EPSRC National Epitaxy Facility and University of Warwick Electron Microscopy Research Technology Platform are also acknowledged for access to equipment used in this work. 


\section{REFERENCES}

[1] Cui, Y., Wei, Q., Park, H. K. and Lieber, C. M. Nanowire "Nanosensors for Highly Sensitive and Selective Detection of Biological and Chemical Species," Science, 293, 1289- 1292, (2001).

[2] Wang, J., Gudiksen, M. S., Duan, X., Cui, Y. and Lieber, C. M. "Highly Polarized Photoluminescence and Photodetection from Single Indium Phosphide Nanowires," Science, 293, 1455- 1457, (2001).

[3] Yan, R., Gargas, D. and Yang, P. "Nanowire photonics," Nat. Photonics, 3, 569- 576, (2009).

[4] Li, K. H., Liu, X., Wang, Q., Zhao, S. and Mi, Z. "Ultralow-Threshold Electrically Injected AlGaN Nanowire Ultraviolet Lasers on Si Operating at Low Temperature," Nat. Nanotechnol. 10, 140-144, (2015).

[5] Zhang, Y., Wu, J., Aagesen, M., Holm, J., Hatch, S., Tang, M., Huo, S. and Liu, H. "Self-Catalyzed Ternary CoreShell GaAsP Nanowire Arrays Grown on Patterned Si Substrates by Molecular Beam Epitaxy," Nano Lett. 14, 4542- 4547, (2014).

[6] Frost, T., Jahangir, S., Stark, E., Deshpande, S., Hazari, A., Zhao, C., Ooi, B. S. and Bhattacharya, P. "Monolithic Electrically Injected Nanowire Array Edge-Emitting Laser on (001) Silicon,” Nano Lett. 14, 4535-4541, (2014).

[7] Zhang, Y., Wu, J., Aagesen, M. and Liu, H. "III-V nanowires and nanowire optoelectronic devices," J. Phys. D: Appl. Phys. 48, 463001(2015).

[8] Qian, F., Li, Y., Gradecak, S., Park, H-G., Dong, Y., Ding, Y., Wang, Z. L. and Lieber, C. M. "Multi-quantum-well nanowire heterostructures for wavelength-controlled lasers," Nat. Mat. 7, 701-706, (2008).

[9] Tatebayashi, J., Ota, Y., Ishida, S., Nishioka, M., Iwamoto, S. and Arakawa, Y. "Highly uniform, multi-stacked InGaAs/GaAs quantum dots embedded in a GaAs nanowire,” Appl. Phys. Lett. 105, 103104 (2014).

[10] Bounouar, S., Elouneg-Jamroz, M., den Hertog, M., Morchutt, C., Bellet-Amalric, E. Andre, R., Bougerol, C., Genuist, Y., Poizat, J.-Ph., Tatarenko, S. and Kheng, K. "Ultrafast Room Temperature Single-Photon Source from Nanowire Quantum Dots,” Nano Lett. 12, 2977-2981, (2012).

[11] Versteegh, M. A. M., Reimer, M. E., Jöns, K. D., Dalacu, D., Poole, P. J., Gulinatti, A., Giudice, A. and Zwiller, V. "Observation of strongly entangled photon pairs from a nanowire quantum dot," Nat. Comm. 5, 5298 (2014).

[12] Heiss, M., Fontana, Y., Gustafsson, A., Wüst, G., Magen, C., O’Regan, D. D., Luo, J. W., Ketterer, B., Conesa-Boj, S., Kuhlmann, A. V., Houel, J., Russo-Averchi, E., Morante, J. R., Cantoni, M., Marzari, N., Arbiol, J., Zunger, A., Warburton, R. J. and Fontcuberta i Morral, A. "Self-assembled quantum dots in a nanowire system for quantum photonics," Nat. Mater. 12, 439-444, (2013).

[13] Yu, Y., Zha, G-W., Shang, X-J., Yang, S. Sun, B-Q., Ni, H-Q., and Niu1, Z-C. "Self-assembled semiconductor quantum dots decorating the facets of GaAs nanowire for single-photon emission." National Science Review 4, 196-209, (2017).

[14] Montinaro, M., Wüst, G., Munsch, M., Fontana, Y., Russo-Averchi, E., Heiss, M., Fontcuberta i Morral, A., Warburton, R. J., and Poggio, M. "Quantum Dot Opto-Mechanics in a Fully Self-Assembled Nanowire.” Nano Lett. 14, 4454-4460, (2014).

[15] de la Mata, M., Zhou, X., Furtmayr, F., Teubert, J., Graděcak, S., Eickhoff, M., Fontcuberta i Morrald, A. and Arbiol, J. "A review of MBE grown 0D, 1D and 2D quantum structures in a nanowire." J. Mater. Chem. C 1, 4300-4312 (2013).

[16] Yu, Y., Dou, X-M., Wei, B., Zha, G-W., Shang, X-J., Wang, L., Su, D., Xu, J-X., Wang, H-Y., Ni, H-Q., Sun, B-Q., Ji, Y., Han, X-D., and Niu, Z-C. "Self-Assembled Quantum Dot Structures in a Hexagonal Nanowire for Quantum Photonics." Adv. Mater. 26, 2710-2717 (2014).

[17] Zhang, Y., Sanchez, A. M., Sun, Y., Wu, J., Aagesen, M., Huo, S. and Liu, H. "Influence of Droplet Size on the Growth of Self-Catalyzed Ternary GaAsP Nanowires," Nano Lett. 16, 1237-1243, (2016).

[18] Zhang, Y., Davis, G., Fonseka, H. A., Velichko, A., Gustafsson, A. Godde, T., Saxena, D., Aagesen, M. Parkinson, P. W. Gott, J. A., Huo, S., Sanchez, A. M., Mowbray, D. J. and Liu, H. "Highly Strained III-V-V Coaxial Nanowire Quantum Wells with Strong Carrier Confinement," ACS Nano, 13, 5931-5938, (2019).

[19] Fonseka, H. A., Velichko, A. V., Zhang, Y., Gott, J . A., Davis, G. D., Beanland, R., Liu, H., Mowbray, D. J. and Sanchez, A. M. "Self-Formed Quantum Wires and Dots in GaAsP-GaAsP Core-Shell Nanowires," Nano Lett. 19, 4158-4165, (2019).

[20] Birner, S., Zibold, T., Andlauer, T., Kubis, T., Sabathil, M., Trellakis, A. and Vogl, P. "Nextnano: General Purpose 3-D Simulations," IEEE T. Electron Dev 54, 2137-2142, (2007).

[21] Zhang, Y., Fonseka, H. A., Aagesen, M., Gott, J. A., Sanchez, A. M., Wu, J., Kim, D., Jurczak, P., Huo, S., and Liu, H. "Growth of Pure Zinc-Blende GaAs(P) Core-Shell Nanowires with Highly Regular Morphology," Nano Lett. 17, 4946-4950, (2017). 
[22] Yan, X., Wei, W., Tang, F., Wang, X., Li, L., Zhang, X., and Ren, X. "Low-threshold room-temperature AlGaAs/GaAs nanowire/single-quantum-well heterostructure laser,” Appl. Phys. Lett., 110(6), 061104. (2017). [23] Stettner, T., Thurn, A., Döblinger, M., Hill, M. O., Bissinger, J., Schmiedeke, P., and Kaniber, M. Tuning “Lasing Emission toward Long Wavelengths in GaAs-(In, Al) GaAs Core-Multishell Nanowires," Nano letters, 18(10), 62926300, (2018).

[24] Alanis, J. A., Saxena, D., Mokkapati, S., Jiang, N., Peng, K., Tang, X., and Parkinson, P. "Large-scale statistics for threshold optimization of optically pumped nanowire lasers," Nano letters, 17(8), 4860-4865, (2017).

[25] Tatebayashi, J., Kako, S., Ho, J., Ota, Y., Iwamoto, S. and Arakawa, Y. "Room-temperature lasing in a single nanowire with quantum dots," Nature Photonics, 9, 501-506 (2015).

[26] Blood, P. “Quantum Confined Laser Devices,” OUP Oxford, 205- 206, (2015).

[27] Finley, J. J., Ashmore, A. D., Lemaitre, A., Mowbray, D. J., Skolnick, M. S., Itskevich, I. E., Maksym, P. A., Hopkinson, M. and Krauss, T. F. "Charged and neutral exciton complexes in individual self-assembled In(Ga)As quantum dots," Phys. Rev. B. 63, 073307 (2001).

[28] Arbiol, J., Magen, C., Becker, P., Jacopin, G., Chernikov, A., Schafer, S., Furtmayr, F., Tchernycheva, M., Rigutti, L., Teubert, J., Chatterjee, S., Morante, J. R. and Eickhoff, M. "Self-assembled GaN quantum wires on GaN/AlN nanowire templates," Nanoscale 4, 7517- 7524, (2012).

[29] Loitsch, B., Winnerl, J., Grimaldi, G., Wierzbowski, J., Rudolph, D., Morkötter, S., Dölinger, M., Abstreiter, G., Koblmüller, G. and Finley, J. J. "Crystal Phase Quantum Dots in the Ultrathin Core of GaAs-AlGaAs Core-Shell Nanowires," Nano Lett. 15, 7544-7551, (2015).

[30] Jeon, N., Loitsch, L., Morkoetter, S., Abstreiter, G., Finley, J., Krenner, H. J., Koblmueller, G. and Lauhon, L. J. "Alloy Fluctuations Act as Quantum Dot-like Emitters in GaAs-AlGaAs Core-Shell Nanowires," ACS Nano. 9, 83358343, (2015). 